\title{
Maternal and neonatal serum lipid levels in preeclamptic females and its correlation with neonatal respiratory distress syndrome \\ B.E.Hassanin ${ }^{1}$, O.A.El Feky ${ }^{1}$, M.E.Fouda ${ }^{2}$,H.A.Torky ${ }^{3}$, M.Bahaa ${ }^{4}$, I.E.Radwan ${ }^{5}$
}

${ }^{1}$ Pediatrics, Dept., Faculty of Medicine, Benha Univ., Benha, Egypt

${ }^{2}$ clinical \&chemical pathology, Dept., Faculty of Medicine, Benha Univ., Benha, Egypt

${ }^{3}$ Gyna \&Obs, Dept., Faculty of Medicine, $6{ }^{\mathrm{TH}}$ October Univ., cairo, Egypt

${ }^{4}$ fellow of peadiatircs ,Dept., Faculty of Medicine, El galaa teaching hospital., cairo, Egypt

${ }^{5}$ peadiatircs specialist Dept., Faculty of Medicine, Shibin Elkom teaching hospital., cairo, Egypt

E-mail: eslameldesoky1980@gmail.com

\begin{abstract}
Background: There have been biological exaggerations of lipid production among women with preeclampsia, but the function of serum lipids in the development of preeclampsia is unclear. In women with preeclampsis, high density lipoprotein (HDL) and LDL have been found to be lower in preeclampsia when plasma concentrations of triglycerides (TG), total cholesterol (TC), low density lipoprotein (LDL) and very low density lipoprotein (VLDL) have been increased. The current research aimed at evaluating maternal and newborn serum lipid levels and their association to neonatal respiratory distress syndrome in preeclamptic females. Methods: This is a case-control research that included two identical groups of preterm infants between 28-34 weeks, with preeclampsia as pre-existing status and normotensive moms. Results: In newborn moms with pre-term labour, RD was shown to be substantially greater than women with pre-eclampsia $(\mathrm{P}<0.05)$. We discovered that newborns with $\mathrm{RD}$ of mothers with pre-eclampsia and mother with child lipid profiles had higher maternal levels of total cholesterol, triglycerides and LDL, but lower levels of HDL, although this variation remained insignificant. The cord level of LDL and triglycerides in the RDS group was nevertheless lower. Conclusion: Maternal lipid levels are an indicator for RDS prediction. In pregnancies complicated by preeclampsia, higher maternal serum lipids have been found. In children and their mothers, RDS is accompanied with lipid changes.
\end{abstract}

Key words: Respiratory distress syndrome, preeclamsia, preterm.

\section{Introduction}

The impact of pre-eclampsia and eclampsia on pregnant mothers, their foetuses, and their infants is catastrophic. The risk of neonatal mortality and increased risk of poor Apgar scores, seize, neonatal encephalopathy and neonatal intense care is about 2 times that of newborn mothers with pre-eclampsia [1].

The primary cause of newborn respiratory failure and mortality, along with hospitalisation in Neonatal Intensive Care Unit (NICU) [2] is NRDS. Neonatal Intensive Distress Syndrome (NRDS) Respiratory distress syndrome (RDS) is one of the most frequent premature birth problems. Preterm RDS is secondary to insufficient surfactants and is typically reciprocally linked to gestational age (GA). There are contradictory facts in the literature on the impact of pre-eclampsia on RDS per se [3].

Lipid metabolism plays a leading role in the late stage of pregnancy foetal growth, including the growth and growth of adipose tissue in foetal intrauterine life [4]. Lipid metabolism also plays a major function in cholesterol transfer in the production of hormones in the surrenal glands of the foetus. It was discovered that higher amounts of amniotic lecithin are linked to respiratory maturation and alterations in phospholipid concentrations in the amniotic fluid [5]. The current research aimed at evaluating maternal and newborn serum lipid levels and their association to neonatal respiratory distress syndrome in preeclamptic females.

\section{Patients and Methods}

The present study included 90 mothers that were subdivided into 2 groups:

- Group 1: which included preeclamptic mothers that gave birth at $28 \& 34$ weeks gestation and their neonates

- Group 2: which included women with preterm labor delivering at the same gestational age (28\&34) and their neonates.

\author{
2.1. Inclusion Criteria \\ Group 1: \\ - Gestational age between 28\&34 weeks. \\ - No other maternal abnormalities. \\ - No fetal abnormalities. \\ Group 2: \\ - Gestational age between 28\&34 weeks. \\ - No other maternal abnormalities. \\ - No fetal abnormalities.
}

2.2. All patients will be subjected to the following The mother

- Full antenatal\& perinatal and natal and postnatal history.

- Dating scan.

- Anomaly scan.

- U/S:within one week before delivery(latest u/s).

- Any additional data were recorded .

The neonate:

- Through clinical examination.

- Blood samples were collected from patients after 12 hours fasting for determination of the serum 
level of triglycerides total cholesterol LDL HDL and VLDL that will be interpreted \& the mean serum lipid concentrations of the two groups will be compared using student's test.

\subsection{Statistical analysis}

The collected data were tabulated and analyzed using SPSS version 24 software (SpssInc, Chicago, ILL Company).Categorical data were presented as number and percentages. Chi square test $\left(\mathrm{X}^{2}\right)$, or Fisher's exact test (FET) were used to analyze categorical variables. Quantitative data were tested for normality using Shapiro-Wilkstest, assuming normality at $\mathrm{P}>0.05$, they were expressed as mean \pm standard deviation, Student " $t$ " test was used to analyze normally distributed variables among 2 independent groups. While non-parametric variables were analyzed using Man Whitney $U$ test. $P$ value $>0.05$ is non-significant (NS) $\mathrm{P}<0.05$ is significant $(\mathrm{S})$.

\section{Results}

By analyzing and processing the data obtained from the history and examination, the study declared that: TG was significantly higher in patients with pre-eclampsia than patients with pre-term labor $(\mathrm{P}<0.05)$. (Table $1 \&$ Fig 1$)$. TC was significantly higher in patients with pre-eclampsia than patients with pre-term labor $(\mathrm{P}<0.05)$ (Table $2 \&$ Fig 2). In the present study we found that HDL was significantly lower in patients with pre-eclampsiathan patients with pre-term labor $(\mathrm{P}<0.05)$ (Table 3$)$. LDL was significantly higher in patients with pre-eclampsia than patients with pre-term labor $(\mathrm{P}<0.05)$ (Fig 3). Cord HDL was lower in pre-eclampsia group that preterm group and both of LDL and triglyceride were higher in pre-eclampsia group that preterm group although this difference still of no significant difference $(P>0.05)$. RD was significantly higher in newborn of mothers with pre-term labor than mothers with pre-eclampsia $(\mathrm{P}<0.05)$ Figure (4). Percentage of SGA was higher in newborn of mothers with pre-eclampsia than mothers with pre-term labor. Newborn with RD had higher maternal levels of total cholesterol, triglycerides and LDL but lower level of HDL although this difference still non significant. However, cord level of LDL and triglycerides were lower in RDS group.

Table (1) Comparison of the TG between patients with pre-eclampsia and patients with pre-term labor

\begin{tabular}{llcccc}
\hline & & $\begin{array}{c}\text { Pre-eclampsia } \\
\mathbf{N}=\mathbf{4 5}\end{array}$ & $\begin{array}{c}\text { Pre-term labour } \\
\mathbf{N}=\mathbf{4 5}\end{array}$ & $\mathbf{X}^{\mathbf{2}}$ & $\mathbf{P}$ \\
\hline & Borderline & $10(22.2 \%)$ & $12(26.7 \%)$ & & 0.005 \\
mother.TG & High & $21(46.7 \%)$ & $7(15.6 \%)$ & $10.782^{\mathrm{a}}$ & \\
& Normal & $14(31.1 \%)$ & $26(57.8 \%)$ & & \\
mother.TG. mg/dL & Mean \pm SD & $198.07 \pm 60.825$ & $158.29 \pm 33.361$ & $\mathrm{t}=3.846$ & $<0.0001$ \\
\hline
\end{tabular}

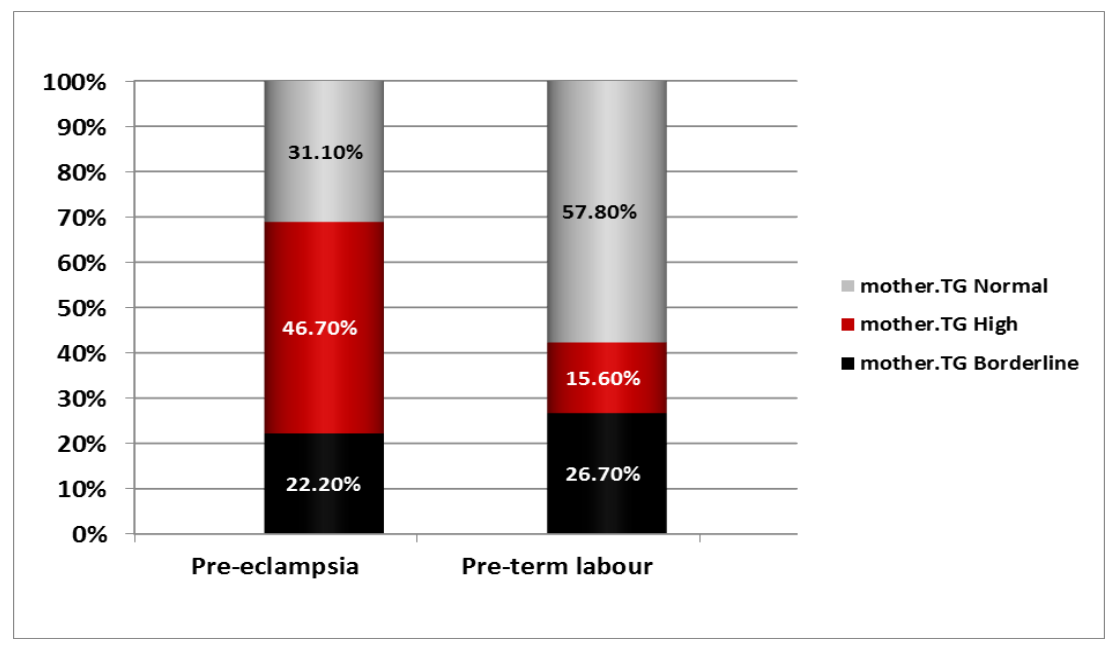

Fig. (1) Bar chart showing comparison of the TG between patients with pre-eclampsia and patients with pre-term labor

Table (2) comparison of the TC between patients with pre-eclampsia and patients with pre-term labor

\begin{tabular}{llcccc}
\hline & & $\begin{array}{c}\text { Pre-eclampsia } \\
\mathbf{N = 4 5}\end{array}$ & $\begin{array}{c}\text { Pre-term labour } \\
\mathbf{N = 4 5}\end{array}$ & $\mathbf{X}^{\mathbf{2}}$ & P \\
\hline \multirow{3}{*}{ mother.TC } & Borderline & $13(28.9 \%)$ & $16(35.6 \%)$ & 11.306 & 0.004 \\
& High & $20(44.4 \%)$ & $6(13.3 \%)$ & & \\
mother.Tc.mg/dl & Mormal & $12(26.7 \%)$ & $23(51.1 \%)$ & & \\
\hline
\end{tabular}




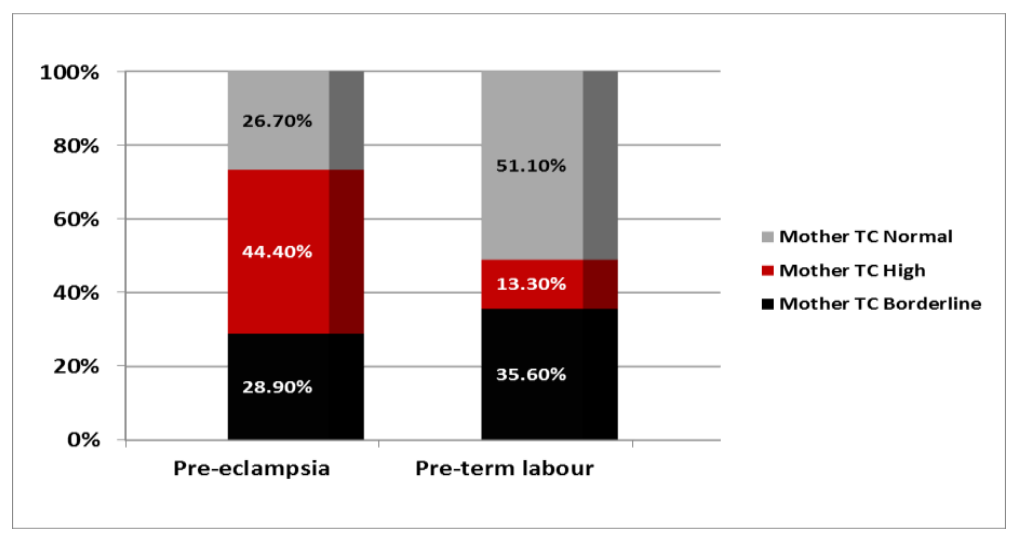

Fig. (2) Bar chart showing comparison of the TC between patients with pre-eclampsia and patients with pre-term labor

Table (3) Comparison of the HDL between patients with pre-eclampsia and patients with pre-term labor

\begin{tabular}{llllll}
\hline & & $\begin{array}{l}\text { Pre-eclampsia } \\
\text { N=45 }\end{array}$ & $\begin{array}{l}\text { Pre-term labour } \\
\text { N=45 }\end{array}$ & $\mathrm{X}^{2}$ & $\mathrm{P}$ \\
& Borderline & $16(35.6 \%)$ & $8(17.8 \%)$ & 6.597 & 0.037 \\
mother.HDL & Low & $12(26.7 \%)$ & $8(17.8 \%)$ & & \\
& Normal & $17(37.8 \%)$ & $29(64.4 \%)$ & & \\
mother.HDL- & Mean \pm SD & $56.36 \pm 14.796$ & $66.62 \pm 16.735$ & $\mathrm{t}=-3.083$ & 0.003 \\
C. $\mathrm{mg} / \mathrm{dL}$ & & & & & \\
\hline
\end{tabular}

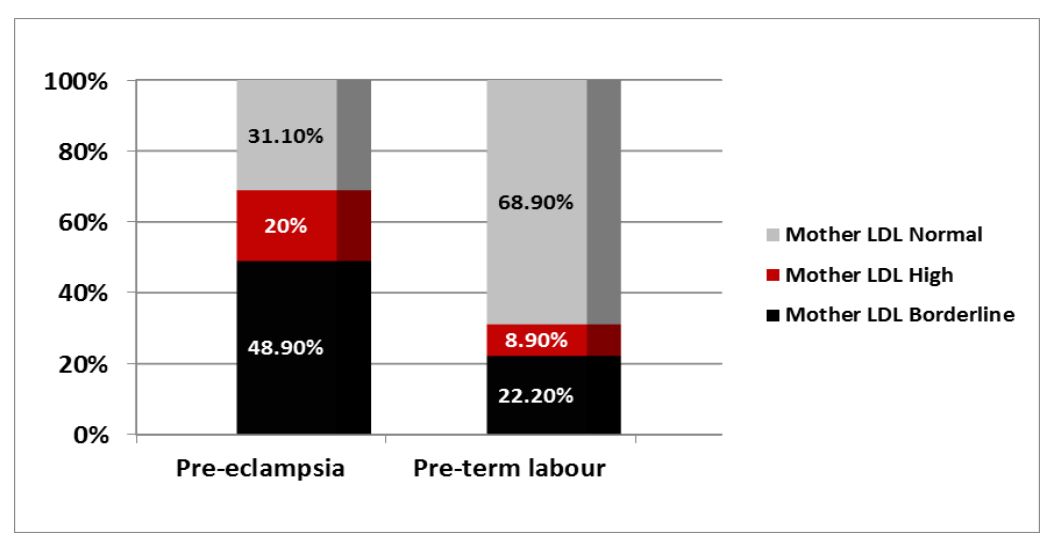

Fig. (3) Bar chart showing comparison of the LDL between patients with pre-eclampsia and patients with pre-term labor

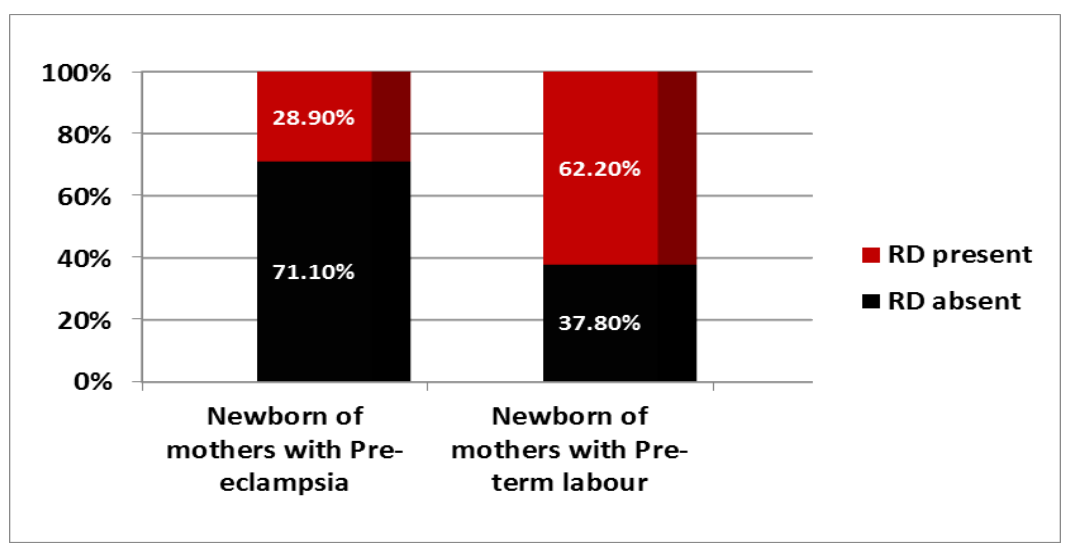

Fig. (4) Bar chart showing comparison of incidence of RD between newborn of mothers pre-eclampsia and mothers with pre-term labor 
Table (4) correlation between the mother TG and the other mother and newborn measurements.

\begin{tabular}{lcc}
\hline & \multicolumn{2}{c}{ mother.TG. mg/dL } \\
& r & P \\
\hline mother.Tc.mg/dl & $0.438^{* *}$ & $<0.0001$ \\
mother.HDL-C. mg/dL & -0.416 & $<0.0001$ \\
mother.LDL-C. mg/dL & $0.590^{* *}$ & $<0.0001$ \\
Age.years & 0.025 & 0.818 \\
Mother weight.Kg & -0.079 & 0.462 \\
systolic.BP & $0.277^{* *}$ & 0.008 \\
Diastolic.BP & $0.299^{* *}$ & 0.004 \\
Newborn weight & -0.115 & 0.282 \\
GA.weeks & -0.168 & 0.113 \\
APGAR.1min & -0.041 & 0.704 \\
APGAR.5min & 0.014 & 0.894 \\
newborn.TC.mg.dl & -0.017 & 0.872 \\
Newborn.HDL.C.mg.dl & -0.111 & 0.299 \\
Newborn.LDL.C.mg.dl & -0.219 & 0.038 \\
Newborn.TG.mg.d & -0.258 & 0.014 \\
\hline
\end{tabular}

\section{Discussion}

In this research TG in patients with pre-eclampsia was considerably greater than in patients with pre-term labour $(\mathrm{P}<0.05)$. Our findings of increased maternal TG in preeclampsia are consistent with many preceding studies that have shown increased lipids in both normal pregnancy and preeclampsia [6].

The mechanism by which hyperlipidemia is caused by pregnancy has not been completely clarified. High maternal triglyceride levels may constitute hyperlipidemia or hyperlipidemia risk. Increased maternal level of TG may have a role in PE pathogenesis and possibly detect pregnant women at risk of preeclampsia and long-term cardiovascular risk [6]. Triglycerides and free fatty acids were previously shown to be higher in pre-eclamptic women in the first and second trimesters [7]. During pregnancy, triglyceride levels also rise in response to action in oestrogen and lipase. In the tissue level, decreased lipoprotein lipase activity and resistance to insulin lead to lower lipid catabolism, which produces hypertriglyceridemia [8]. However, several earlier researches should be noted, which did not indicate significant changes in lipid parameters in both groups $[9,1]$.

In this research, the TC was much greater in preeclampsia patients than in pre-term labour patients $(\mathrm{P}$ $<0.05)$. The investigations performed in China and India has shown similar results [11, 12]. During pregnancy the level of plasma cholesterol rises in response to an increased oestrogen driven lipoprotein synthesis or a failure to remove lipoprotein lipids. For placental steroid and Placental membrane-synthesis, higher plasma cholesterol levels are utilised and stored as a maternal fat reserve that acted as fuel both for the mother and the developing baby later in pregnancy or lactation[13].

In the current research we discovered that HDL in pre-eclampsiathan individuals with pre-term labour was substantially lower $(\mathrm{P}<0.05)$. The research performed [12, 14] showed similar results. HDL transports cholesterol from peripheral tissues to the liver, which is separated for excretion and utilised for biomolecular synthesis. Higher levels of HDL lipoprotein are protective against high blood pressure and cardiovascular disease [15].

In the current research, LDL in patients with preeclampsia was substantially greater than in prelaboratory individuals $(\mathrm{P}<0.05)$. Evidence has also been revealed in an earlier study [16]. The pathophysiology of pre-eclampsia is associated with increased lipid oxidation products and reduced antioxidant levels [17]. Various reporting has shown that in females with higher oxidised low density lipoprotein (LDL) and triglyceride (TG) levels of circulating vitamin $\mathrm{C}$ compared to normal intensive pregnant women the risk of pre-eclampsia increases [18]. The oxidative conversion of LDL-cholesterol to LDL oxidised forms is the essential event for atherosclerosis and hypertension start and progression [19].

In the current research, we observed that in the pre-eclampsia group, the cord HDL was lower, while both the preterm group and the LDL and triglyceride were greater than the pre-eclampsia group while there was no significant difference $(\mathrm{P}>0.05)$.

Ophir and his colleagues found no such connection and only saw modest rise in LDL-C in preeclamptic pregnancy UCB [20], while Rodie and colleagues noted substantial increases in the TG, TC and TC/HDL-C ratios of preeclamptic women's foetuses in $\mathrm{UCB}[21]$.

A theoretical explanation given by Romanowicz and Bańkowski indicated the buildup cholesterol in the umbilical cord wall may result in "early ageing" of the umbilical cord tissue, thereby triggering the development of hypertension.

We observed in the current research that RD was considerably greater in pre-term moms than in preeclampsia $(\mathrm{P}<0.05)$. An earlier research has shown a 
reduced incidence of RDS in babies with preeclampsia mothers; it was hypothesised that in the preeclampsia group more protective variables existed than in the control group [3]. Maternal preeclampsia has been shown to have a protective impact against RDS, and foetal lung maturity has been accelerated [23].

In the current research, we discovered that SGA is greater in pre-eclampsia moms than in preterm mothers. A recent research has shown that the status of SGA is a frequent pre-eclampsia complication with a widespread 13,36-52 percent incidence[24]. Although the link between preeclampsia and severe RDS in their subgroup analysis had been negligible in an earlier research, the trend towards higher risk in both categories remained continuous. This result shows that preeclampsia and SGA do not interact substantially with RDS. In the whole dataset, 95 percent of CIs for the combination of preeclampsia and severe RDS have been extremely near to 1 , which may explain why a substantial difference in the analytics in the subgroup of this small sample has been not detected [3].

Newborns with RD exhibited greater maternal concentrations of total cholesterol, triglycerides and LDL but lower HDL while the difference was still not significant. The cord level of LDL and triglycerides in the RDS group was nevertheless lower. In neonates with no RDS and in the patients with RDS, the latest research revealed an important direct connection between gestational age and triglyceride and cholesterol levels [25, 24].

Fetal development relies directly on the nutrients that go through the placenta to the infant. Mother adjusts her metabolism to the constant supply and bodily requirements of nutrients [26].

Bansal and his colleagues have shown that the placenta may be passed through by maternal cholesterol and that its mother concentration influences the neonate level [27]. Pregnancy and delivery factors, as well as specific conditions, may affect the metabolism of foetal and neonatal lipids. Cholesterol synthesis inhibition results in poor surfactant production. Premature babies with RDS were shown to have substantially lower amounts of cord lipid serum than normal term infants. Although only preterm infants have been included in the current research and the differences in cord serum lipid levels have not been documented [28]. Furthermore, low cord serum lipids limit foetal development in the utero, and may delay the ripening of the foetal lungs [26]. Placental transportation of lipid components in preterm babies who had RDS may be proposed to be abnormal [28].

\section{Conclusion}

Maternal levels of lipids are a guide for the development of peredict RDS. In pregnancies affected by preeclampsia, higher maternal serum lipids have been observed. In children and their mothers, RDS is accompanied with lipid changes. Moms who had RDS with pre-Eclampsia exhibited moderate dyslipedemia.

\section{References}

[1] P.von Dadelszen, D.Sawchuck, G.Justus Hofmeyr, LA.Magee, H.Bracken, M.Mathai. PRE-EMPT (PRE-eclampsia-Eclampsia Monitoring, Prevention and Treatment): A low and middle income country initiative to reduce the global burden of maternal, fetal and infant death and disease related to preeclampsia. Pregnancy Hypertens.vol.3(4),pp.199202,2013.

[2] P.Rijal, M.Shrestha Scenario of neonatal respiratory distress in tertiary hospital.Birth.vol.14(10),pp.6-12,2018.

[3] YH.Wen, HI.Yang, HC.Chou. Association of Maternal Preeclampsia with Neonatal Respiratory Distress Syndrome in Very-LowBirth-Weight Infants. Sci Rep.vol.9,pp.132140,2019.

[4] Q.Kan, S.Ding, Y.Yang, X.Zhou Expression profile of plasma microRNAs in premature infants with respiratory distress syndrome. Molecular medicine reports.vol.12 (2),pp.2858-2864,2015.

[5] P.Green, M.Theilla, and P.Singer Lipid metabolism in critical illness. Current Opinion in Clinical Nutrition and Metabolic Care.vol.19(2),pp.111-115,2016.

[6] T.I.Alahakoon, H.J.Medbury, H.Williams. Lipid profiling in maternal and fetal circulations in preeclampsia and fetal growth restriction-a prospective case control observational study. BMC Pregnancy Childbirth.vol.20,pp.61-70,2020.

[7] E.Gratacos Lipid-mediated endothelial dysfunction: a common factor to preeclampsia and chronic vascular disease. Eur J Obstet Gynecol Reprod Biol.vol.92(1),pp.63-6,2000.

[8] E.Herrera, G.Desoye Maternal and fetal lipid metabolism under normal and gestational diabetic conditions. Horm Mol Biol Clin Investig.vol.26(2),pp.109-127,2016.

[9] J.Chalas, F.Audibert, J.Francoual, Le B.Bihan, R.Frydman, A.Lindenbaum Concentrations of apolipoproteins E, C2, and C3 and lipid profile in preeclampsia. Hypertens Pregnancy.vol.21,pp.199-204,2002.

[10] DJ.Vanderjagt, RJ.Patel, AU.El-Nafaty, GS.Melah, MJ.Crossey, RH.Glew High-density lipoprotein and homocysteine levels correlate inversely in preeclamptic women in Northern Nigeria. Acta Obstet Gynecol Scand.vol.83,pp.536-42,2004.

[11] CN.Spracklen, CJ.Smith, AF.Saftlas, JG.Robinson, KK.Ryckman Maternal hyperlipidemia and the risk of preeclampsia: a meta-analysis. American journal of epidemiology.vol.180(4),pp.346-358,2014.

[12]E.Jasovic-Siveska, D.Milkovski Maternal plasma lipid concentration in first and second trimester of pregnancy and risk of 
preeclampsia. Obstetrics \& Gynecology International Journal.vol.10(4),pp.36-70,2019.

[13] A.Basaran Pregnancy-induced hyperlipoproteinemia: review of the literature. Reproductive sciences (Thousand Oaks, Calif).vol.16(5),pp.431-437,2009.

[14]AR.Avidime, MA.Tella, G. Hadiza, OO.Abiodun A comparative Study of Serum Lipid Levels in PreEclamptic and Normotensive Pregnant Women in a Tertiary Hospital, Northwest Nigeria. Biomed J Sci \& Tech Res.vol.3(1),pp.3003-3011,2018.

[15]A.Flaquer, S.Rospleszcz, $\quad$ E.Reischl, S.Zeilinger, H.Prokisch, T.Meitinger. Mitochondrial GWA Analysis of Lipid Profile Identifies Genetic Variants to Be Associated with HDL Cholesterol and Triglyceride Levels. PloS one.vol.10(5),pp.155-180, 2015.

[16] KN.Choudhury,

AK.Mainuddin, M.Wahiduzzaman, Islam SM: Serum lipid profile and its association with hypertension in Bangladesh. Vascular health and risk management.vol.10,pp.327-332,2014.

[17] S.Gupta, N.Aziz, L.Sekhon, R.Agarwal, G.Mansour, J.Li. Lipid Peroxidation and Antioxidant Status in Preeclampsia A Systematic Review. Obstetrical and gynecological survey.vol. 64(11),pp.750$759,2009$.

[18]S.Timalsina, P.Gyawali, A.Bhattarai Comparison of lipid profile parameters and oxidized low-density lipoprotein between normal and preeclamptic pregnancies in a tertiary care hospital in Nepal. Int $\mathbf{J}$ Womens Health.vol.8,pp.627-631,2016.

[19]C.Qiu, TTT.Phung, S.Vadachkoria, M.MuyRivera, SE.Sanchez, MA.Williams: Oxidized Low-Density Lipoprotein (Oxidized LDL) and the Risk of Preeclampsia. Physiol Res.vol.55,pp.491-500,2006.

[20]E.Ophir, G.Dourleshter, Y.Hirsh, V.Fait, L.German, Bornstein J. Newborns of preeclamptic women: a biochemical difference present in utero. Acta Obstet Gynecol Scand.vol.85(10),pp.1172-1178,2006.

[21] VA.Rodie, MJ.Caslake, F.Stewart. Fetal cord plasma lipoprotein status in uncomplicated human pregnancies and in pregnancies complicated by pre-eclampsia and intrauterine growth restriction. Atherosclerosis.vol.176(1),pp.181$187,2004$.

[22]L.Romanowicz, E.Bańkowski Preeclampsiaassociated alterations in sphingolipid composition of the umbilical cord artery. Clin Biochem.vol.42(16-17),pp.1719-1724,2009.

[23] J. Langenveld. Neonatal outcome of pregnancies complicated by hypertensive disorders between 34 and 37 weeks of gestation: a 7 year retrospective analysis of a national registry. Am J Obstet Gynecol.vol. 205(540),pp.541-547,2011.

[24] N.Tul. Outcome of small for gestational age preterm singletons: a population-based cohort study. J Perinat Med.vol.44,pp.941-944,2016.

[25] R.Kelishadi. Comparison of Serum Triglyceride and Cholesterol Levels in Premature Neonates with or without Respiratory Distress Syndrome (RDS) International Journal of Pediatrics,Article ID .vol.15,pp.177-190,2021.

[26]RN.Sehra, A.Garg, Verma S.Umbilical Cord Serum Lipid Profile of Normal Preterm Neonates and Preterm Neonates with Respiratory Distress Syndrome: A Hospitalbased Cross-sectional Study. Indian Journal of Neonatal Medicine and Research.vol.8(4),pp. 34-38,2020.

[27] N.Bansal, JK.Cruickshank, P.McElduff, PN.Durrington Cord blood lipoproteins and prenatal influences. Curr Opin Lipidol.vol.16(4),pp.400-08,2005.

[28] DM.Lane, WJ.McConathy, MA.McCaffree, M.Hall Cord serum lipid and apolipoprotein levels in preterm infants with the neonatal respiratory distress syndrome. J Matern Fetal Neonatal Med.vol.11(2),pp.118-25,2002. 